TRETJAKOFF, D. K. (1916).-The sense organs of Lampetra fluviatilis (in Russian). Bull. Physico-Math. Dept. Imp. Novoross. Univ., Odessa.

VoG'T, C., und YUNG, E. (1894).--Lehrbuch der praktischen vergleichenden Anatomie. Bd. II. Braunschweig: Vieweg.

WALLS, G. L. (1928a).-An experimental study of the retina of the brook lamprey, Entosphenus appendix (De Kay). Journ. Comp. Neurol., Vol. XLVI, pp. 465-473. $(1928 b)$. - The photo-mechanical changes in the retina of mammals. Science. Vol. LXVII, pp. 655-656.

(1932).-The hot celloidin technic for animal tissues. Stain Technol., Vol. VII, pp. 135-145.

(1934a). The reptilian retina. I. A new concept of visual-cell evolution. Amer. Journ. Ophthal., Vol. XVII, pp. 892-915.

(1934b).-Human rods and cones. The state of knowledge. Arch. of Ophthal., Vol. XII, pp. 914-930.

- and JUDD, H. D. (1933). - The intra-ocular colour-filters of vertebrates. Brit. Journ. of Ophthal., Vol. XVII, pp. 641-675 and 705-725.

Woollard, H. H. (1927). The differentiation of the retina in the Primates. Proc. Zool Soc., London, 1927, pp. 1-17.

\title{
SOME NOTES ON THE TREATMENT OF STRABISMUS*
}

\author{
BY \\ SANFORD R. GIFFORD, M.D. \\ FROM THE DEPARTMENT OF OPHTHALMOLOGY, NORTHWESTERN \\ UNIVERSITY MEDICAL SCHOOL, CHICAGO, ILLINOIS
}

IT is the object of these remarks to outline a routine of treatment which has proved, in my hands and those of the group working in our clinic, very satisfactory. Many will differ with me on the details of operative procedure, for there are a number of operations for strabismus, each of which will give good results in the hands of those familiar with it. On the other phases of treatment, however, it seems that we should agree fairly well. After our two years' experience with a special clinic for orthoptic training I think we may set down certain definite facts as to the possibilities and limitations of such training, and give it its definite place in the treatment of strabismus.

The first step in any case is, of course, a complete examination, including refraction under atropine in children under 10 years of age. In older children homatropine is usually as effective. At least our retinoscopy under homatropine will tell us whether cycloplegia is complete and in a few cases will indicate the need for atropine refraction.

In concomitant convergent squint with hyperopia or hyperopic astigmatism as much of the full correction as will be tolerated is

* Read at the Pacific Coast Oto-Ophthalmologic Society, Butte, Montana, July, 1934. 
prescribed. If the full correction reduces vision too much, the correction prescribed will be increased to the full correction as rapidly as possible.

Cases with less than one dioptre of hyperopia offer less chance of correction with glasses alone, but may show some improvement with bifocals, or grab fronts, +2.0 D. sph. being added for near. It is also worth trying this in cases which are straight for distance but show $10^{\circ}$ to $15^{\circ}$ of convergence when reading. It is hard to estimate what proportion of cases will become straight with glasses, since the chance of this depends on the age at which treatment is begun, the degree of refractive error and other factors. Dr. Guibor's ${ }^{1}$ control series, observed very carefully after refraction and with no other treatment except atropine or occlusion, included cases of concomitant squint of all types and degrees, in which refraction was done quite early, usually below the age of six years. After six months 12.5 per cent. had become straight, while after 18 months 30 per cent. were straight with glasses. Twenty per cent. remained straight for brief periods without glasses. Most of these were, of course, cases of concomitant convergent squint.

In divergent squint, little is to be expected of correcting the refractive error, as a rule. Non-operative measures must here be confined to attempts to improve amblyopia and to fusion training.

With regard to the improvement of vision in amblyopic eyes, opinions vary widely. Those who practise prolonged uni-ocular occlusion with a fixed dressing for weeks and months report the best results. Delord ${ }^{2}$ by covering one eye for from four to six months obtained improvement of vision in 35 cases. One improved from $1 / 10$ to $2 / 10$, one from $1 / 50$ to $3 / 10$, and one from $2 / 50$ to $5 / 10$. Herzau ${ }^{3}$, from the Leipzig clinic, reports 37 per cent. of cases with definitely improved vision.

Peter $^{4}$ reported that 50 per cent. showed improvement.

Our results with amblyopia have been far below those just mentioned. It has been impossible to obtain co-operation for prolonged uni-ocular occlusion in most cases. It is impossible as a rule where the vision of the amblyopic eye is below 20/200, and only a few parents will carry it out satisfactorily in other cases. In several cases, however, in which co-operation was excellent the results were disappointing.

With occlusion for one or two hours a day and the use of atropine results have not been at all remarkable in our hands.

Dr. Guibor has used atropine a great deal and also a system of reducing vision in the good eye by under-correction with atropine or the use of Snell's lined glasses. This is of value in overcoming suppression of the poorer eye and as an aid to orthoptic training, but actual improvements in vision have not 
been striking. In cases with less than $20 / 200$ vision he saw no improvement. A few cases with vision of $20 / 100$ improved to $20 / 80$. Ten cases with vision of $20 / 50$ were recorded as showing $\frac{}{0}$ improvement, sometimes to $20 / 20$, but these were chiefly children three to four years of age in whom the initial vision could hardly be reliable. One is inclined to think of possible errors in recording $\stackrel{\mathbb{\Omega}}{\propto}$ vision in the series of authors reporting such marked improvement \& as is done by Herzau and Peter. But it must be said that we have

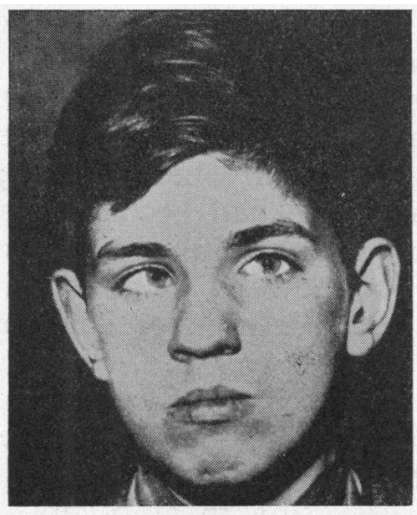

FIG. 1

Case 1.-Convergent strabismus before training.

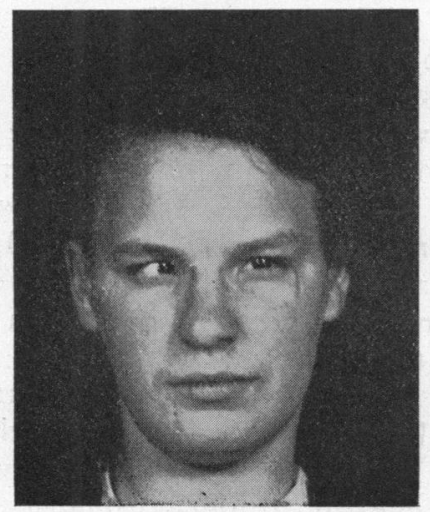

FIG. 3.

Case 2. Convergent strabismus before training.

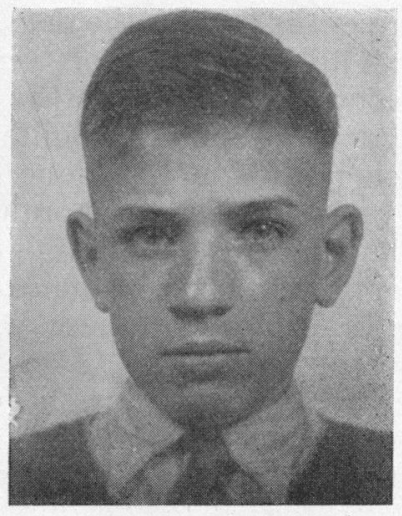

FIG. 2.

Case 1. After three months orthoptic training.

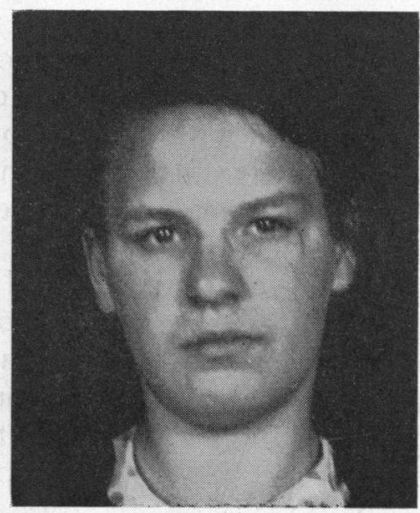

Fig. 4.

Case 2. After one year orthoptic training.

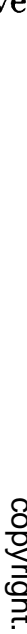


not given prolonged uni-ocular occlusion a fair and prolonged trial in a large enough number of cases to form a definite opinion. As the lower limit of vision with which orthoptic training could be carried out, Dr. Guibor found $20 / 80$ in most cases. Patients with poorer vision could be trained to fuse the larger test objects, but did not obtain much benefit from the exercises. This agrees fairly well with the opinion of Cantonnet ${ }^{5}$ who is most enthusiastic as to the results of orthoptic training in most cases.

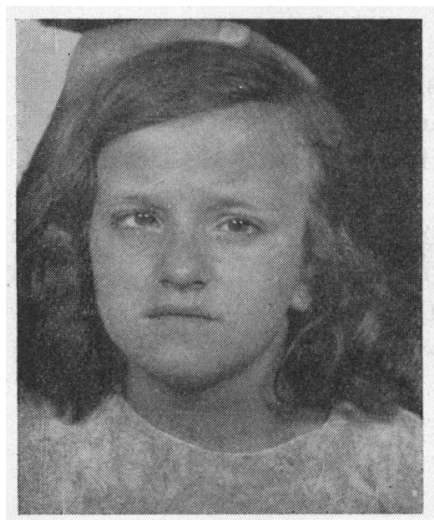

FIG. 5.

Case 3. Convergent strabismus before training.

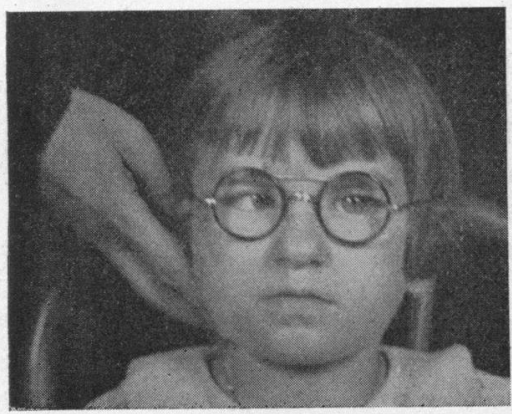

Fig. 7.

Case 4. Convergent strabismus before training.

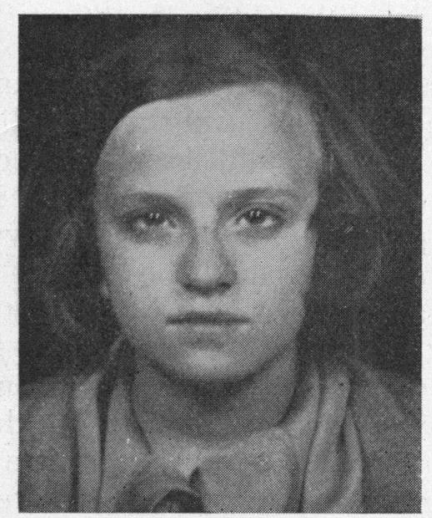

FIG. 6.

Case 3. After nine months orthoptic training.

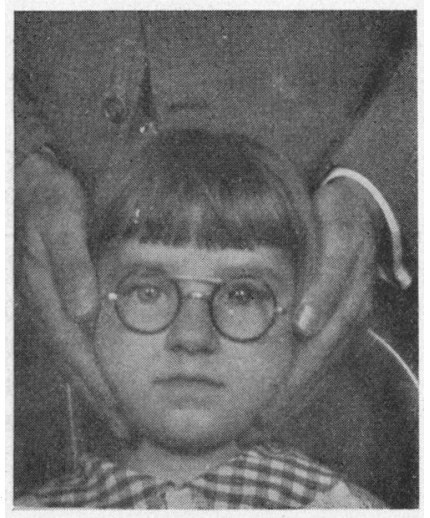

Fig. 8.

Case 4. After six months orthoptic training. 
In attempting to decide which cases are suitable for orthoptic training or other measures, Guibor classifies his cases. First paralytic squint must be ruled out, a proceeding not always perfectly easy with small children. These cases, especially of abducens palsy, are now considered suitable for surgery at the outset, since many good results may be obtained with tendon transplantation. Next to be ruled out are cases of pseudo-strabismus, patients with a wide or narrow angle gamma. Measurement of the angle gamma and the cover test are all that is necessary.

Most important is the group of accommodative strabismus. These cases have hyperopia, usually of fairly high degree, and show a marked improvement in the angle of squint under atropine. Many become straight on full refractive correction and atropine. The use of additional correction for reading is here often of assistance. Fusion training seems to be of value in hastening the process, but is not always necessary. This class is a large one and furnished the greater part of the 30 per cent. in Guibor's control series which became straight without any training.

Strabismus due to defective fusion is a class necessary to explain those cases with good vision and little or no refractive error, which show no change in the angle of squint under atropine. Many cases of divergent strabismus are of this type, especially the cases of intermittent or latent divergence. Cases of alternating squint are used as the type of this group, and such cases are stated by Peter and other authors to have complete absence of the fusion sense. Although nearly all cases of squint have difficulty in fusion and may be unable to fuse when first seen-most cases acquire this ability very rapidly. In three cases of alternating squint we have been unable to develop any fusion ability. In four equally typical cases, however, good fusion and depth perception have been developed. In two of these, of $25^{\circ}$ and $10^{\circ}$, the squint has been corrected with glasses and training. In the two others the angle has remained unimproved in spite of good fusion in the stereoscope. Hence, while we may say that the fusion ability is under-developed in such cases, it cannot be stated that it is absent. This agrees with what we know of the anatomy and physiology of vision, as expressed by Adler6. It is in this group that fusion training is most important. In it improvement may be obtained in most cases and full correction in a number of cases of low or moderate degree. Where surgery becomes necessary, fusion training before-and especially afteroperation will greatly enhance the benefits of surgery and do much to insure their permanency.

Strabismus with amblyopia.-Whether amblyopia is the cause of strabismus or vice versa in this group, it is certain that the results of refraction are less satisfactory in proportion to the degree 
of amblyopia and that where vision is less than $20 / 100$ or $20 / 80$, fusion training is of little value. Attempts will be made to improve the amblyopia, without, however, expecting too much, and if hyperopia is present, the effect of glasses for six months to a year will be watched. After this time, there is no reason for postponing surgery.

Strabismus associated with anisometropia may be considered a variety of the above group if the anisometropia is of high enough degree to prevent binocular vision with correction. It is possible to attain binocular vision in some such cases by means of the iseikonic lenses of Ames which equalize the size of the retinal images. As far as I know, no cases in which squint has been corrected by their use have been reported.

Strabismus due to muscular abnormalities may be used to describe cases in which none of the above factors is present and in which tests reveal a weakness or over-action of certain muscles. In convergent squint of this type uni-ocular abduction can be maintained for only a short time without difficulty, as shown by nystagmoid movements. In cases of vertical strabismus where no paresis can be demonstrated, a muscular anomaly must always be assumed to exist. In this group, surgery is indicated without preliminary training. A trial of other measures may be necessary before certain cases can be placed in this group.

Cutting across this classification, but equally important, is a division of all types into squint of high and low degree. Guibor found that in his group of 48 cases, including all types of concomitant strabismus, where the angle of squint was $15^{\circ}$ or less, 84 per cent. became straight after refraction and orthoptic training, while where the angle was more than $15^{\circ}$, only 16 per cent. became straight. In this latter group, therefore, it is often more practical to advise surgery without a too prolonged trial of glasses and training. The operation should be followed by training which can be much more easily conducted when the eyes are straight or nearly so. I have been especially gratified with the effect of orthoptic training in a small group of cases with $5^{\circ}$ or $10^{\circ}$ of squint remaining after operation. It has been my experience that such cases usually became worse in the days when no post-operative training was attempted. For a large part of this group, however, in which training was carried out immediately after operation, the remainder of squint was seen to disappear completely within two to three months, and the eyes have remained straight.

There is only time to outline the methods of training which have proved of value in the clinic. I am borrowing freely from Dr. Guibor for this. Patients come once a week and are given training for 20 minutes to an hour. In this clinic group, usually no home 
training was conducted, for practical reasons. In private practice, where parents are co-operative, training is done chiefly at home, with office visits seldom oftener than once a month, at which progress is checked and further work outlined. Usually additional prisms are prescribed for use during the exercises. Where co-operation at home is not good, more frequent office visits should be made. One is inclined, however, not to insist on many office visits, for fear patients will feel that the training is being erected into a racket. Hence clinic patients will, on the whole, be given a fairer trial of training than private patients. At the first visit a careful record is made of the degree of squint, and fusion ability.

The suppression usually present is the first thing to overcome. For this the large synoptophore of Maddox is ideal, since the light may be cut down before the fixing eye till the squinting eye alone is able to see its chart. Then the light is increased before the fixing eye till both charts are seen. They may be brought together and fused at the first visit. The same effect may be obtained by placing a very bright light before one tube of Worth's amblyoscope or by using larger objects before the squinting eye in the stereoscope. Prisms are usually necessary to approximate the images. These will be base out for convergent and base in for divergent squint. As soon as fusion with the stereoscope iso possible exercises are conducted with this instrument since more stereoscopes and a greater variety of charts are available. We have found no real advantage in the more complicated instruments employing moving objects.

In nearly all cases with vision of $20 / 100$ or better in the squinting eye, fusion is possible with the simpler instruments with the aid of prisms. These are used in grab-fronts or placed in holders in the stereoscope. The stereoscope itself has prisms which allow the correction of 5-7 degrees of deviation at the extremes of its slide.

It has been found that the easiest charts to fuse are not the flat pictures, such as the bird and the cage, but simple figures showing definite perspective in which one or more parts are common to both pictures. Such are the "E" series of Wells and all of the Sattler charts. Guibor has just prepared a set of charts for children and adults all of which have perspective and are graduated in order of difficulty. They are split charts, like the Sattler charts, hence possessing two advantages over the Wells charts, which have both pictures printed on one chart. The split charts may be reversed, thus reversing the depth impression so that it may not be learned by children. They may also be placed at any distance apart in the special holder, so that each pair of pictures may be used in all the positions of the Wells " $\mathrm{H}$ " and "I" series, which saves much changing of prisms and often 
obviates their use at the beginning. A number of different sets of charts are advisable, to vary the exercises and prevent children from learning what they are supposed to see. A number of pictures are kept out as check charts, so that they can never be learned, and provide a check on suppression and depth perception. It is surprising how rapidly depth perception is obtained in children who have squinted for years, and we agree with Cantonnet that persons aged 25 or 30 years may obtain it. A number of such

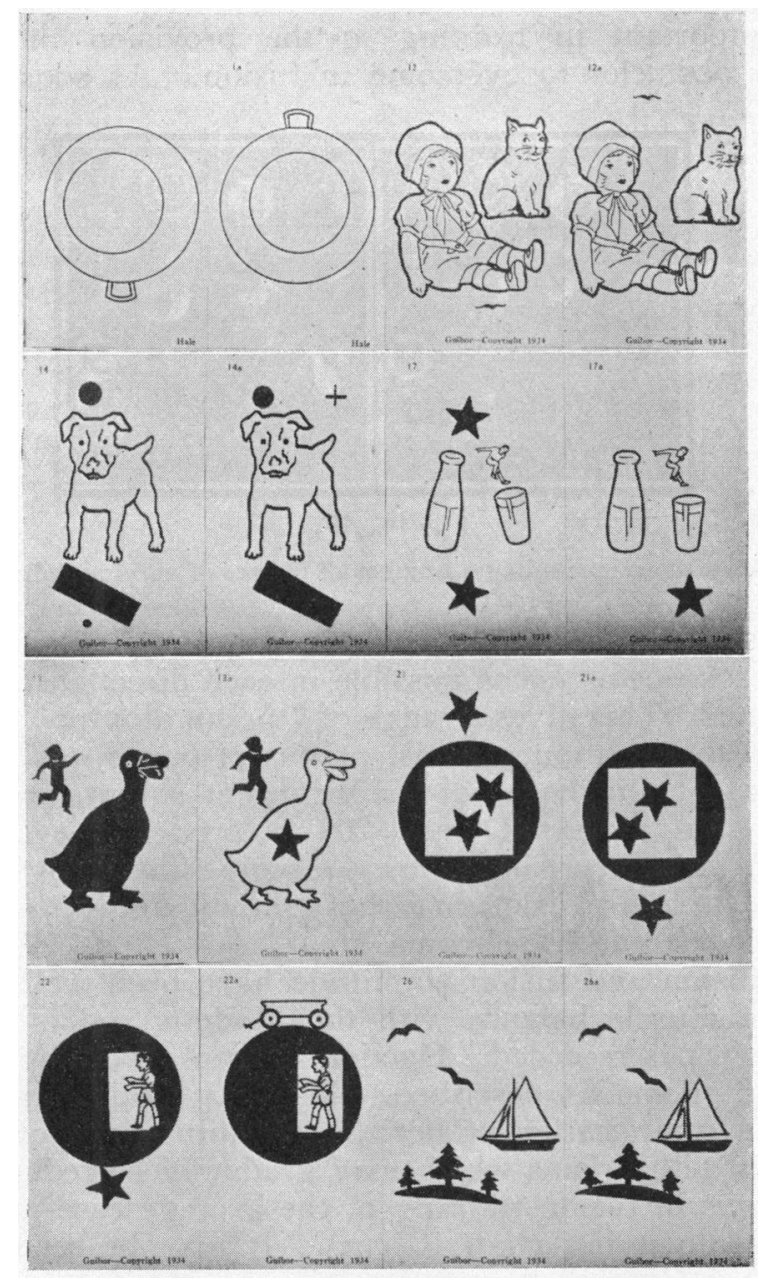

Fig. 9.

Group of Guibor's charts, pairs of split charts of increasing difficulty. 
patients have been checked with the Howard apparatus, and there is no question that true binocular depth perception is present. $\Rightarrow$ We have learned to watch for pseudo-binocularism, the condition in which, by rapid alternating movements, both charts are seen and appear to be superimposed. This is learned by watching the $\frac{\bar{\sigma}}{\overline{0}}$ patient's eyes in the stereoscope for which purpose the top of $\bar{\pi}$ the stereoscope shield should be cut away. A number of patients go through a stage of pseudo-stereoscopic vision in which the depth impression is seen reversed. This usually yields rapidly to true stereopsis and depth perception.

Most important in training is the provision of constantly increasing obstacles to overcome in fusion. As soon as objects $\frac{0}{\circ}$

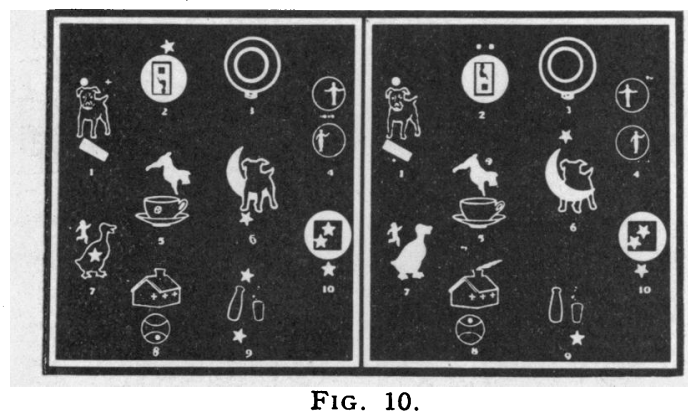

Review chart, including a number of figures of varying difficulty.

can be fused, fusion amplitude is developed by moving the arm $\stackrel{\varnothing}{\stackrel{\circ}{\circ}}$ of the stereoscope as far as possible in each direction while fusion $\overrightarrow{\vec{B}}$ is maintained. 'This gives a range of 7 prism dioptres. When this 9 is accomplished without prisms, prisms of opposite sign, base in for convergent and base out for divergent squint, are provided, and these are increased until $20^{\circ}$ before each eye are overcome. It is surprising how rapidly the original prism necessary to obtain fusion may be reduced, and opposite prisms of increasing strength overcome.

When fusion and fusion amplitude have been well developed, a check of muscle balance with the Maddox rod is made, and 9 duction power is recorded. Duction is then developed further by the use of prisms as described. When a vertical imbalance is found as a complication of horizontal squint, it is often well to $N$ correct this with prisms which may gradually be reduced.

The stages in fusion training of the average case may be seen ${ }_{N}^{N}$ in the accompanying chart (Chart). It may be seen that most progress is made in the first four to six months, if co-operation has been good and that only slight additional improvement may be expected after this time. 


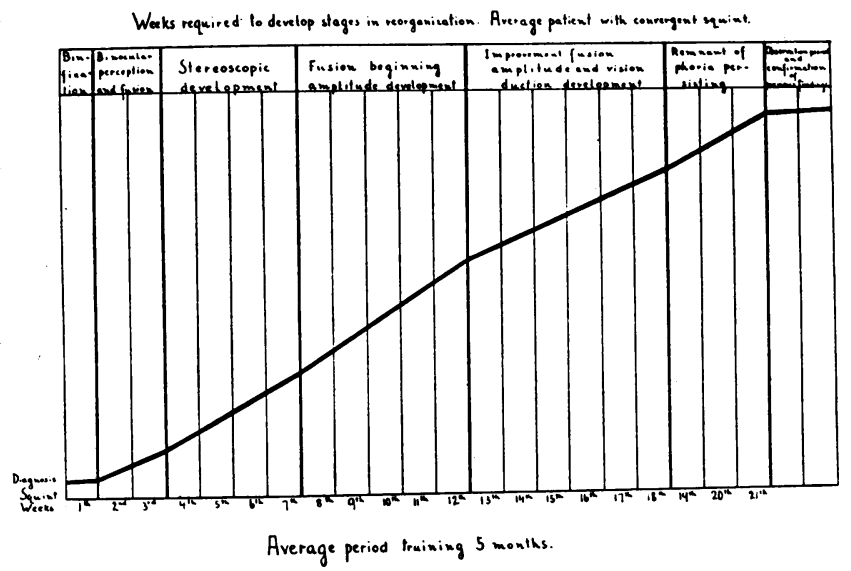

FIG. 11.

Stages in orthoptic training.'

As to the age when operation should be performed, I agree with Peter that when a fair trial of non-operative measures has been given and further progress is at a stand-still, operation should be advised, regardless of age. It is a great pity to have any child get to the age at which it is sensitive, self-conscious and set apart from its fellows by such a ludicrous deformity which is so easily remediable.

In discussing operative procedures, it would not be profitable to describe in detail anyone's favourite operation. No one operation is suitable to all cases of squint, and the important thing to have is a reasonable plan of the procedures or combinations of such which work out best in particular types or degrees of squint. Theoretically, we should classify our cases into those due to overaction or insufficiency of a muscle-group and treat them by respectively lengthening or shortening the offending muscle. But practically in nearly every case of concomitant strabismus we have a combination of both factors, insufficiency of one muscle followed by spasm of its opponent or vice versa. This is a theoretical reason, but I would like to point out other reasons for a procedure which we have come to adopt in nearly all cases of concomitant squint higher than $20^{\circ}$. I refer to simultaneous operation on both lateral recti of one eye. First of all, many patients with an amblyopic eye refuse to have any operation on their good eye so that what is done must be done to the amblyopic eye. Second and most important, a much greater effect can be obtained from an advancement, for example, if it is combined with a recession than when it is first done alone and followed later by recession. 
If one is afraid of tension on the advancement sutures there would seem to be less danger of this after weakening the opposing muscle at the time of the advancement. Thirdly, the effect on the antero-posterior position of the eye and hence on the width of the palpebral fissure of any squint operation must be considered. If one should correct a $35^{\circ}$ convergent squint by one advancement and resection, the eye would necessarily be pulled back in the orbit a millimetre or more. This would narrow the palpebral fissure and give the appearance of partial ptosis. If both external recti are advanced to a less degree, both eyes will be pulled back slightly and both palpebral fissures become narrower. I think we have all seen the opposite effect in patients who have had complete tenotomy of one internal rectus allowing the eye to come forward and turn out. Obviously the only method which will not change the antero-posterior position of the eye is an operation on both lateral recti which simply turns the eye around without pulling it in either direction.

When the angle of squint is large, or even over $25^{\circ}$, I prefer a resection and advancement combined with a very moderate recession. This never interferes with convergence for practical purposes, and with it one can correct at least $35^{\circ}$ and sometimes $45^{\circ}$ in a single operation. Rather than run the risk of convergence insufficiency the recession is never more than $4 \mathrm{~mm}$., and if any squint remains, it can be cared for by a later recession on theo opposite eye. Since beginning orthoptic training at once in such cases, a second operation has less often been necessary.

I have no particular technical tricks about the advancement operation, except that a slight simplification of the Worth suture has seemed quite effective and that no attempt is made to close the conjunctiva with the advancement sutures. An untied running conjunctival suture is employed, which is especially convenient to remove in children. Removal of the deep sutures is apt to be troublesome, and in children it is best to give a few whiffs of gas for this purpose.

Recession is done by two methods. When general anaesthesia must be employed, one must estimate beforehand the amount of recession to be done and do it without regard to the position of the eyes under the anaesthetic. In this, Jameson's method is ideal, but I employ two double-armed sutures, and only pass one needle of each pair through the scleral fibres. When operating under local anaesthesia, one will often prefer a type of recession which may be varied until the desired position is obtained. The operation is really a guarded tenotomy which has been described by many authors, and recently by Mott ${ }^{7}$, and Wilkinson..$^{8 .}$

In a case of convergent squint, for example, the internal rectus is first exposed, clamped, and cut from its insertion. Two double- 


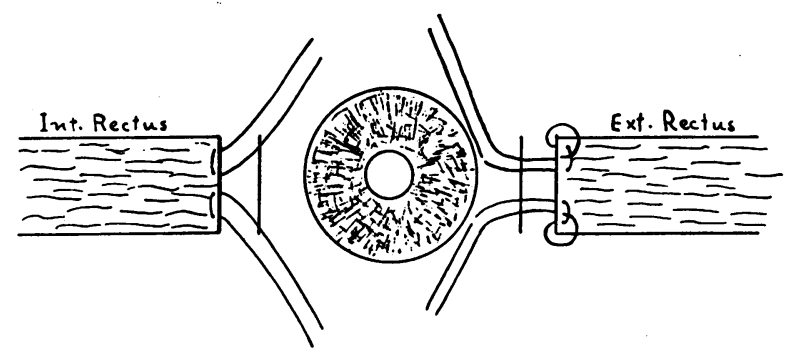

FIG. 12.

Technique of guarded tenotomy combined with advancement.

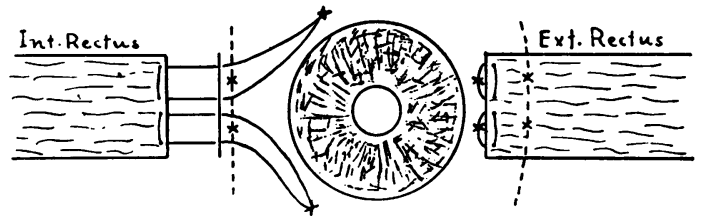

FIG. 13

Technique of guarded tenotomy combined with advancement.

armed sutures are placed through the tendon, the forceps is released and the advancement on the opposite rectus is performed. Then the sutures through the internal rectus are placed through the insertion and conjunctiva and given one turn of the knot. This is then tightened until the desired position is obtained when the knot is tied. It is impossible for the muscle to slip back further, and only occasionally does the effect turn out to be less than desired. This may be due to a return of function in a muscle partially paralyzed by novocaine, or the muscle may become attached further forwards than the position obtained on the operating table which is, of course, prevented by the Jameson technique. In some cases, as in recession of a vertical muscle, I have prevented this slipping forward by a suture placed around the tendon and with one bite through the sclera beneath it when the desired position was obtained.

In squint of slight degree I was trained to employ tucking by the Banister-Valk technique. There were some disappointments, even in such cases, and a tendency in a few cases for the squint to recur after an apparently perfect result. Then there was always the hump which required months or years to disappear. And in a few cases the buried catgut remained unabsorbed and formed small granulomata requiring later removal. So recession has been more and more employed in such cases, and with very satisfactory results. Here and in the phorias the O'Connor operation seems to have a very definite place. Certainly it appears to be the safest 
and surest form of tucking. I have been a little hesitant to use it in many cases on account of the unsightly reactions which I have seen, but one cannot talk to Californians without a renewed decision to give it a fair trial in cases where a moderate shortening is indicated. It seems impossible, however, that it can replace advancement where a large effect is desired. O'Connor often reinforces its effect by central tenotomy of the opposing muscle, but this has always seemed much less certain and accurate than recession. Certainly, O'Connor's fear of causing convergence insufficiency by a recession, whatever his models may show, is not borne out by practical experience with many recessions on the human eye, it being understood that the operation is carefully performed, and that the muscle is never set back more than $5 \mathrm{~mm}$.

\section{LITERATURE}

1. Guibor.-Arch. of Ophthal., Vol. II, p. 433, 1934.

2. Delord.-Arch. d'Ophtal., Vol. XXXVIII, p. 597, 1921.

3. Herzau.-Klin. Monatsbl. f. Augenheilk., Vol. XC. p. 551, 1933.

4. Peter.-Amer. Jl. of Ophthal., Vol. XVI, p. 481, 1933.

5. Cantonnet et al.-Le Strabisme. Paris, 1932.

6. Adler.-Ocular Physiology. 1933.

7. Mott.-Arch. of Ophthal., Vol. VIII, p. 858, 1932.

8. Wilkinson.-Arch. of Ophthal., Vol. XI, p. 471, 1934.

\section{FAMILIAL MACULAR DEGENERATION}

BY

Lieut.-Col. R. E. Wright, C.I.E., I.M.S.

SUPERINTENDENT, GOVERNMENT OPHTHALMIC

HOSPITAL, MADRAS

From time to time during the past 15 years macular degenerations have been met with here, in the course of routine fundus examinations, which presented certain peculiar similarities in appearance. It has been customary to use expressions such as "beaten brass or silver," "coppery sheen," etc. to describe the ill-defined macular fundus changes seen. I have for long regarded some of these cases as familial degenerations, as on rare occasions such appearances have been met with in more than one member of the same family. Moreover, they did not resemble any fundus change, with which we were familiar, connected with known pathological states, except, perhaps, the ill-defined changes which are sometimes seen after the resolution of bilateral subhyaloid haemorrhages. Owing to the difficulty which exists in obtaining accurate family histories from our South Indian patients, striking sibships are rarely forthcoming, but a family group met with 